\title{
ESTUDO MORFOMÉTRICO DE Gliricola quadrisetosa Ewing, 1924 RECUPERADO EM Galea spixii Wagler, 1831
}

\author{
Josivania Soares Pereira ${ }^{1}$, Janilene de Oliveira Nascimento ${ }^{2}$, Kalianne Carla de Sousa \\ Aguiar $^{2}$, Moacir Franco de Oliveira ${ }^{3}$, Wesley A. Costa Coelho ${ }^{4}$, Sílvia Maria Mendes \\ AHID $^{3}$
}

${ }^{1}$ Pós-Graduanda da Universidade Federal Rural do Semi-Árido, Mossoró, RN, Brasil - josigej@ufersa.edu.br

${ }^{2}$ Graduandas em Medicina Veterinária da Universidade Federal Rural do Semi-Árido, Mossoró, RN, Brasil.

${ }^{3}$ Professores Doutores da Universidade Federal Rural do Semi-Árido, Mossoró, RN, Brasil.

${ }^{4}$ Professor Doutos da Faculdade de Enfermagem Nova Esperança, Mossoró, RN, Brasil

\begin{abstract}
O estudo morfométrico de piolhos que acometem Galea spixii auxilia na compreensão da taxonomia desse Phithraptera. O trabalho objetivou realizar a morfometria de espécimes de Gliricola quadrisetosa recuperados em G. spixii. As análises foram realizadas com auxílio de microscópio óptico de luz. Os dados foram apresentados na forma de média simples e desvio padrão. Os machos e fêmeas apresentaram um comprimento médio corporal de $98,01 \pm 2,43$ e $117,97 \pm 4,75 \mu \mathrm{m}$; da face inferior da cabeça de $16,42 \pm 3,41$ e $18,43 \pm 2,61 \mu \mathrm{m} ; \quad$ do protórax de $12,43 \pm 0,91$ e $13,79 \pm 1,13 \mu \mathrm{m}$ e do pterotórax de $13,57 \pm 1,07$ e $14,88 \pm 1,84 \mu \mathrm{m}$, respectivamente. A
\end{abstract}

\section{RESUMO}

PALAVRAS-CHAVE: Ectoparasito, piolho, taxonomia. genitália do macho apresentou em sua placa basal comprimento respectivo da margem lateral externa e interna de $28,86 \pm 1,29$ e $21,88 \pm 1,91 \mu \mathrm{m}$; do ramo externo

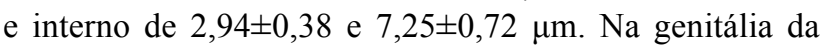
fêmea, o comprimento da porção superior e inferior foi respectivamente de $25,35 \pm 2,32$ e $28,18 \pm 2,86 \mu \mathrm{m}$. As 11 cerdas apresentaram comprimento médio de $2,99 \pm 0,17$ $\mu \mathrm{m}$. As medidas obtidas contribuirão para padronização da taxonomia de Gliricola quadrisetosa recuperados em Galea spixii.

\section{MORPHOMETRIC STUDY OF Gliricola quadrisetosa Ewing 1924, RECOVERED IN \\ Galea spixii Wagler, 1831}

\section{ABSTRACT}

The morphometric study on lice affecting Galea spixii helps in understanding the taxonomy of this Phithraptera. The study aimed to perform morphometry of Gliricola quadrisetosa specimens recovered in G. spixii. Analyses were performed using light optical microscope. Data were presented as simple average and standard deviation. Males and females showed an average length of body of 98,01 2,43 and $117,97 \pm 4,75 \mu \mathrm{m}$; from the underside of the head $16,42+3,41$ and $18,43 \pm 2,61 \mu \mathrm{m}$; prothorax $12,43 \pm$ 0,91 and $13,79+1,13 \mu \mathrm{m}$ and pterothorax $13,57 \pm 1,0 \overline{7}$ and $14,88 \pm 1,84 \mu \mathrm{m}$, respectively. The male genitalia lodged in their basal plate showed length of its external 
and internal lateral margin of 28,86+1,29 and 21,88 \pm $1,91 \mu \mathrm{m}$; external internal branch of $2,94 \pm 0,38$ and 7,25 $\pm 0,72 \mu \mathrm{m}$. In the female genitalia, the length of the upper and lower portions were, respectively, 25,35 $\pm 2,32$ and

KEYWORDS: Ectoparasite, lice, taxonomy.

\section{INTRODUÇÃO}

A morfometria constitui o estudo da forma e do tamanho e de como essas duas variáveis se relacionam entre si. Trata-se de uma maneira de analisar os parâmetros corporais que podem auxiliar na verificação do dimorfismo sexual das espécies ${ }^{1}$. Permite também avaliar a variação intraespecífica, bem como a interespecífica e é um aspecto importante no diagnóstico característico dos organismos. Pelo estudo morfométrico, é possível observar as variações no tamanho de estruturas consideradas importantes para taxonomia dos seres vivos e, dessa forma, auxiliar na classificação e diagnóstico de espécies parasitárias que acometem os animais selvagens e domésticos ${ }^{2}$.

Dentre os animais selvagens, Galea spixii (Wagler, 1831) é um pequeno roedor de pelagem densa e híspida pertencente à Subordem Hystricognathi, Família Caviidae e Subfamília Caviina $^{3,4}$. É comumente encontrado em todos os estados da região Nordeste e também em Minas Gerais e Mato Grosso ${ }^{5,6}$.

Quando em cativeiro, os preás podem ser acometidos por ectoparasitos, dentre os quais os piolhos, correspondentes à espécie Gliricola quadrisetosa Ewing, 1924, que interferem na saúde e bem-estar destes cavídeos por ocasionar prurido intenso, alopecia e crostas cutâneas na pele, predispondo à infecção bacteriana secundária ${ }^{7}$.

O estudo taxonômico de Gliricola quadrisetosa recuperados em pequenos roedores mamíferos foi descrito recentemente por Pereira ${ }^{7}$, que o descreveram como Amblycera com protórax arredondado, meso e metatórax fundidos (pterotórax), placa esternal com aspecto circular e côncava. As fêmeas diferenciam-se dos machos pela genitália e por apresentar como característica única e marcante desta espécie a presença de dois pares de longas cerdas localizadas no $2^{\circ}$ e $3^{\circ}$ pleurito abdominal.

Apesar do conhecimento de algumas características morfológicas que auxiliam na identificação de G. quadrisetosa, existe uma
$28,18 \pm 2,86 \mu \mathrm{m}$. The 11 bristles had an average length of $2,99 \pm 0,17 \mu \mathrm{m}$. The measurements will help to standardize the taxonomy of Gliricola quadrisetosa recovered in Galea spixii.

escassez de dados na literatura quanto à padronização da taxonomia deste Phthiraptera. Objetivou-se realizar a morfometria de algumas características morfológicas de espécimes machos e fêmeas de $G$. quadrisetosa recuperados em $G$. spixii cativos do Rio Grande do Norte, Brasil.

\section{MATERIAL E MÉTODOS}

Utilizaram-se 50 exemplares de Gliricola quadrisetosa, sendo 25 machos e 25 fêmeas, pertencentes ao acervo do Laboratório de Parasitologia Animal (LPA) da Universidade Federal Rural do Semi-Árido (UFERSA). Os piolhos foram recuperados por penteamento da área corporal dorsal de Galea spixii, cativo do Centro de Multiplicação de Animais Silvestres (CEMAS) da UFERSA, localizada na cidade de Mossoró/RN, autorizado para criação com fins científicos pelo IBAMA (Instituto Brasileiro do Meio Ambiente e dos Recursos Naturais Renováveis) sob número de registro 12.492-004.

Os ectoparasitos mantidos no LPA em frascos com álcool a 70\% foram transferidos para placas de Petri contendo solução de hidróxido de potássio a $10 \%$ para realização do processo de clareamento. Posteriormente, os exemplares foram colocados entre lâmina e lamínula para morfometria, realizada com auxílio de microscópio óptico de luz em objetiva de 10X e 40X. As medidas obtidas em cada ocular micrométrica foram multiplicadas por fator de correção, sendo 1,36 para objetiva de $10 \mathrm{X}$ e 0,33 para objetiva de $40 \mathrm{X}$.

Foram determinados o comprimento corporal, face inferior da cabeça, protórax e pterotórax. Nos exemplares fêmeas mediram-se as cerdas presentes no $2^{\circ}$ e $3^{\circ}$ pleuritos abdominais.

Realizaram-se delimitações de algumas áreas a serem medidas na genitália de ambos os sexos: o comprimento das margens laterais externa e interna e dos ramos externo e interno nos machos; e o comprimento da porção superior e inferior, além do comprimento das 11 cerdas presentes nos gonopodos e porção inferior nas fêmeas (Figura 1). 

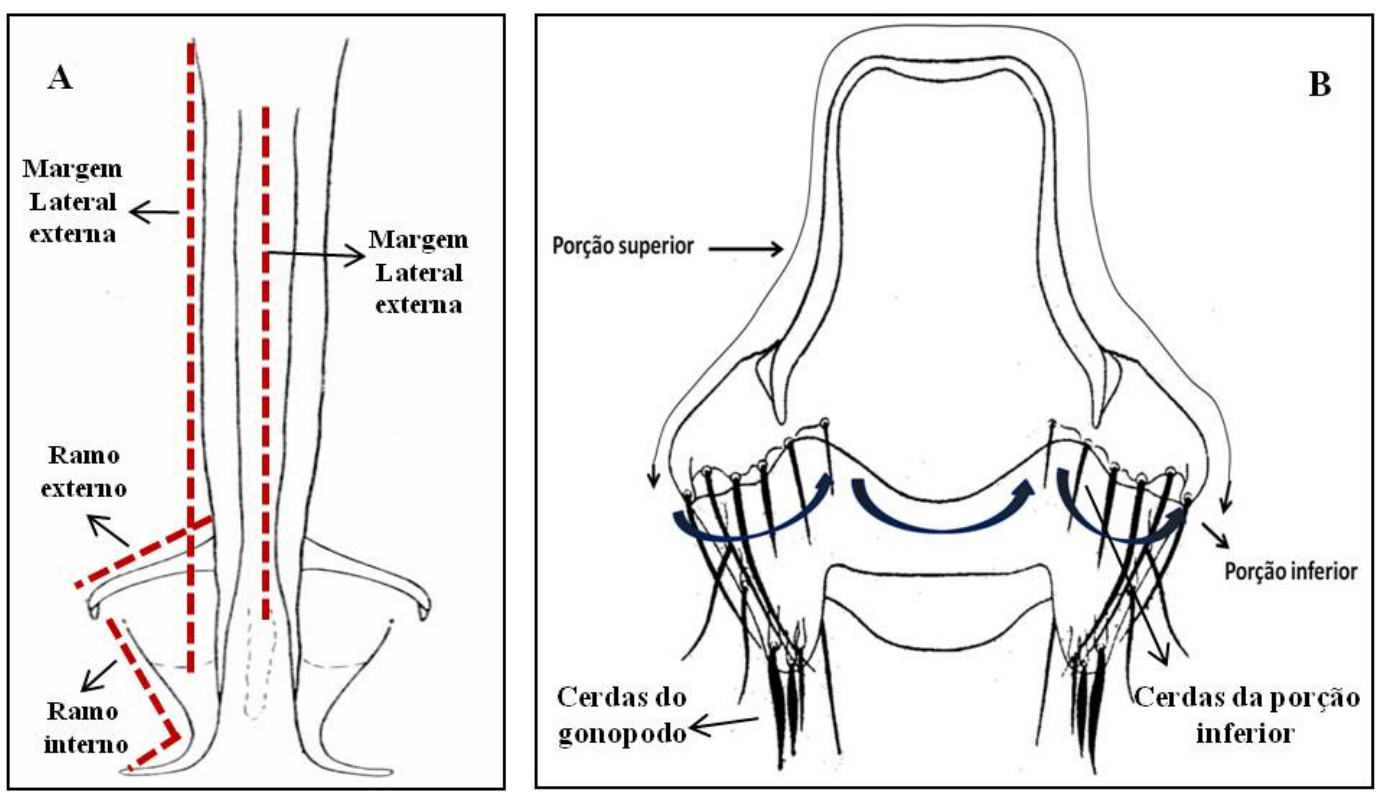

Figura 1 - Áreas delimitadas para morfometria da genitália de G. quadrisetosa. A- Macho; B- Fêmea. Adaptação de Werneck (1936) (7).

Os dados foram apresentados na forma de média e desvio padrão. A comparação estatística dos dados foi realizada por meio do Teste de MannWhitney. O nível de significância foi estabelecido como $\mathrm{p}<0,05$. As análises foram realizadas com auxílio do programa BioEstat 5.0.

\section{RESULTADOS E DISCUSSÃO}

A partir da morfometria realizada, observouse que todos os valores médios das medidas das fêmeas foram superiores aos dos machos (Tabela 1).

Tabela 1 - Medidas das características morfométricas da espécime Gliricola quadrisetosa, de acordo com o sexo

\begin{tabular}{lcccc}
\hline Sexo & Comprimento corporal & $\begin{array}{c}\text { Face inferior da } \\
\text { cabeça }\end{array}$ & Protórax & Pterotórax \\
\hline Macho & $98,01 \pm 2,43 \mathrm{~b}$ & $16,42 \pm \underline{3,41 \mathrm{~b}}$ & $12,43 \pm 0,91 \mathrm{~b}$ & $13,57 \pm 1,07 \mathrm{~b}$ \\
Fêmea & $117,97 \pm 4,75 \mathrm{a}$ & $18,43 \pm 2,61 \mathrm{a}$ & $13,79 \pm 1,13 \mathrm{a}$ & $14,88 \pm 1,84 \mathrm{a}$
\end{tabular}

Em cada morfometria (coluna), médias seguidas de letras diferentes apresentam diferença estatística significativa entre si pelo teste de Mann-Whitney $(\mathrm{p}<0,05)$.

Morfometria em G. quadrisetosa foi recentemente realizada por Pereira et al. ${ }^{8}$ e Pereira et al. ${ }^{9}$. Medidas de comprimento total do corpo deste ectoparasito foi anteriormente realizada por Werneck $^{10}$, que registrou para um espécime fêmea coletado em Cavia porcellus comprimento de 1,25 $\mathrm{mm}$ e para um macho, $0,88 \mathrm{~mm}$. Este fato corrobora os dados obtidos na presente pesquisa na qual se observou que os machos apresentaram menor tamanho em relação às fêmeas.

Quanto à realização da morfometria na genitália dos machos e fêmeas do Phthiraptera estudado no presente trabalho, estas medidas foram obtidas pela primeira vez, sendo observado que, nos machos, a placa basal apresentou comprimento respectivo da margem lateral externa e interna de 
$28,86 \pm 1,29$ e $21,88 \pm 1,91 \mu \mathrm{m}$; e do ramo externo e interno de $2,94 \pm 0,38$ e 7,25 $\pm 0,72 \mu \mathrm{m}$ (Figura 2 ).
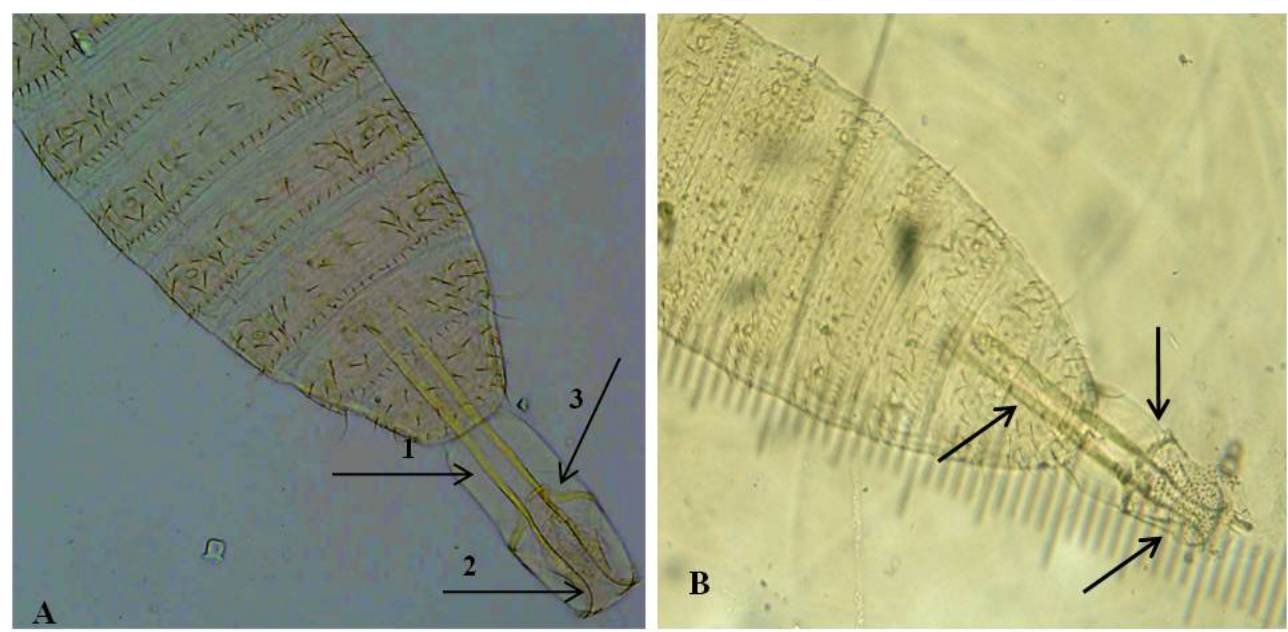

Figura 2- Genitália do macho de Gliricola quadrisetosa. A- Margem lateral externa indicada através da seta 1; ramo interno destacado através da seta 2; ramo externo indicado através da seta 3 (objetiva 10X). BMedidas da genitália indicada através das setas (objetiva 10X).

Já para a genitália da fêmea, o comprimento da porção superior e inferior foi, respectivamente, de $25,35+2,32$ e $28,18+2,86 \mu \mathrm{m}$. Já as 11 cerdas presentes na porção inferior e nos gonopodos apresentaram comprimento respectivo de: $1,71+$ 0,$26 ; 2,19+0,48 ; 2,72+0,67 ; 3,83+0,86 ; 4,31+$ 0,$76 ; 4,04+0,77 ; 3,15+0,67 ; 3,07+0,68 ; 2,77+$ 0,$51 ; 2,71 \pm 0,44 ; 2,39 \pm 0,51$ (Figura 3 ).

Como as cerdas do $2^{\circ}$ e $3^{\circ}$ pleurito abdominal são consideradas, segundo Werneck ${ }^{10}$ e Pereira et al. ${ }^{8}$, uma característica marcante nesta espécie, uma vez que somente neste piolho é que elas são observadas, fez-se pela primeira vez a morfometria das mesmas, sendo que a primeira, indicada através do número um, apresentou 14,39+ $2,78 \mu \mathrm{m}$ e a segunda, indicada pelo número dois, teve $15,81 \pm 2,26 \mu \mathrm{m}$ de comprimento (Figura 4).

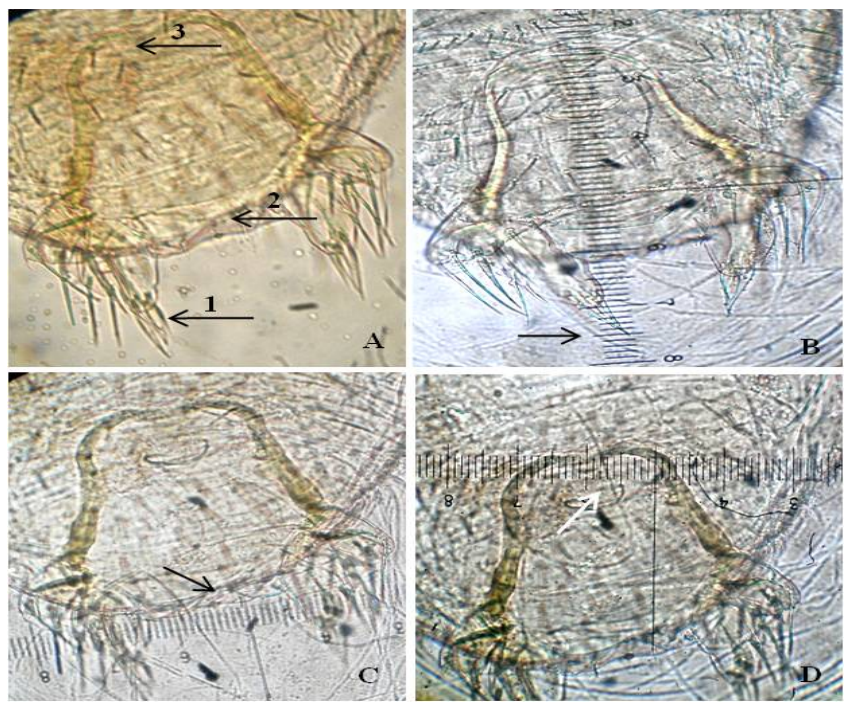

Figura 3- Genitália da fêmea de Gliricola quadrisetosa. A- Cerdas dos gonopodos indicadas através da seta 1 ; porção inferior indicada através da seta 2; porção superior indicada através da seta 3 (objetiva40X). BMedida das cerdas dos gonopodos (objetiva 40X). C- Medida da porção inferior (objetiva 40X). Medida da porção superior (objetiva 40X). 


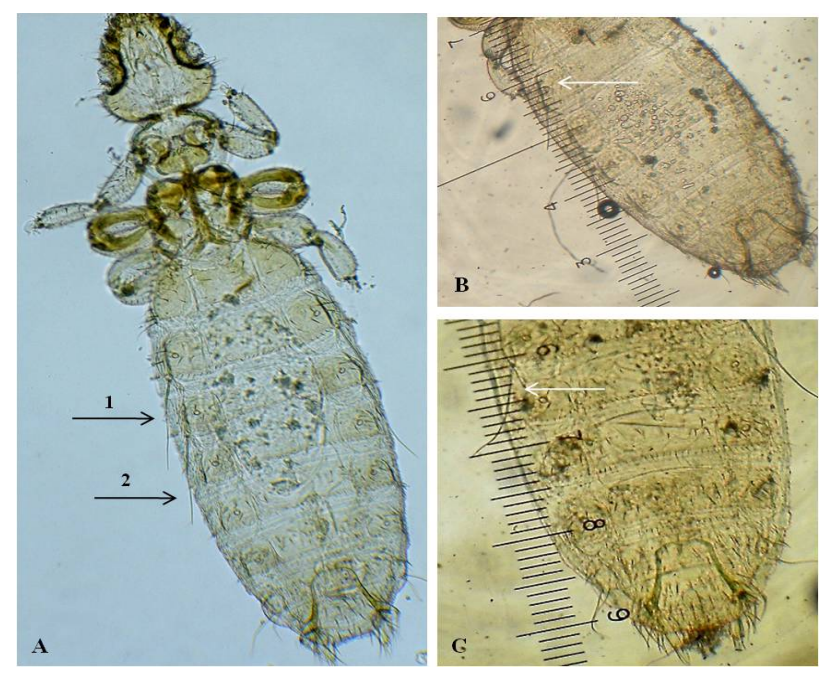

Figura 4- Fêmea de Gliricola quadrisetosa. A- Cerda do $2^{\circ}$ pleurito indicada pela seta 1 ; cerda do $3^{\circ}$ pleurito indicada através da seta 2 (objetiva 10X); B- Medida da cerda do $2^{\circ}$ pleurito (objetiva 10X); C- Medida da cerda do $3^{\circ}$ pleurito (objetiva 10X).

\section{CONCLUSÕES}

As características morfológicas medidas em Gliricola quadrisetosa auxiliam na padronização da taxonomia desta espécie de piolho, bem como na classificação e diferenciação de outras espécies de Phthiraptera que acometem o Galea spixii. O presente trabalho relatou ainda pela primeira vez a realização de medidas das cerdas presentes no $2^{\circ}$ e $3^{\circ}$ pleurito abdominal, característica única e marcante desta espécie de piolho. Além de notificar medidas da genitália do macho e da fêmea, nunca antes descritas.

\section{REFERÊNCIAS}

1. Machado LF, Paresque R, Christoff AU. Anatomia comparada e morfometria de Oligoryzomys nigripes e $\mathrm{O}$. flavescens (Rodentia, Sigmodontinae) no Rio Grande do Sul, Brasil. Papéis Avulsos de Zoologia. 2011; 51(3):

2. Gibbons LM. Revision of the genus Haemonchus Coob, 1988 (Nematoda: Trichostrongylidae). Systematic Parasitology. 1979; 1: 3-24.

3. Barbosa PBBM, Queiroz PVS, Jerônimo SMB, Ximenes MFFM. Experimental infection parameters in Galea spixii (Rodentia: Caviidae) with Leishmania infantum chagasi. Memórias do Instituto Oswaldo Cruz. 2008; 103:545-548.

4. Bonvicino CR, Oliveira JA, D'Andrea PS. Guia dos roedores do Brasil, com chaves para gêneros baseadas em caracteres externos. Rio de Janeiro: Centro PanAmericano de Febre Aftosa - OPAS/OMS. . 2008;120p. Disponível em http://www.fiocruz.br/ioc/media/livro\%20roedores.pdf.

5. Moojen J. Os Roedores do Brasil. Rio de Janeiro: Ministério de Educação e Saúde. Instituto Nacional do Livro; Biblioteca Científica Brasileira; 1952. (Série A, v.2).

6. Lacher JR, T.E. The comparative social behavior of Kerodon rupestris and Galea spixii and the evolution of behavior in the Cavidae. Bulletin of Carnegie Museum of Natural History. 1981; 17:1- 71.

7. Pereira JS, Carvalho LCA, Soto-Blanco B, Oliveira MF, Ahid SMM. Ectoparasitos em preás (Galea spixii Wagler, 1831) cativos no semiárido do Rio Grande do Norte. Pesquisa Veterinaria Brasileira. 2012; 32 (8): 789793.

8. Pereira JS, Nascimento JO, Aguiar KCS, Oliveira MF, Ahid SMM. Estudo morfométrico da placa esternal de Gliricola quadrisetosa Ewing, 1924 recuperados em Galea spixii Wagler,1831. Pubvet, Londrina. 2011;, 5 (38), ed. 185, Art. 1247.

9. Pereira JS, Melo AEC, Sousa FSR, Oliveira MF, Ahid SMM. Parasitismo por Gliricola quadrisetosa Ewing, 1924 em Galea spixii wagler, 1831: redescrição da placa esternal. Acta Veterinaria Brasilica. 2012; 6 (1): 45-47.

10. Werneck FL. Contribuição ao conhecimento dos Mallophagos encontrados nos mamíferos sul-americanos. Memórias do Instituto Oswaldo Cruz. 1936; 31: 391-589. 